\title{
The Internal Cerebral Vein: New Classification of Branching Patterns Based on CTA
}

\author{
(D). Brzegowy, (D) M.P. Zarzecki, (D) A. Musiał, (D) H.M. Aziz, (D)T. Kasprzycki, (D)R.S. Tubbs, (D)T. Popiela, and (D).A. Walocha
}

\begin{abstract}
BACKGROUND AND PURPOSE: The internal cerebral vein begins at the foramen of Monro by the union of the thalamostriate and the anterior septal veins. The lateral direct vein is its other major tributary. Numerous researchers have reported differences in internal cerebral vein branching patterns but did not classify them. Hence, the objectives of this study were to evaluate the anatomy of the internal cerebral vein and its primary tributaries and classify them depending on their course patterns using CTA.
\end{abstract}

MATERIALS AND METHODS: Head CTAs of 250 patients were evaluated in this study, in which we identified the number and termination of the anterior septal vein and the lateral direct vein. The course of the lateral direct vein and its influence on the number of thalamostriate veins and their diameters and courses were assessed. The anterior septal vein-internal cerebral vein junctions and their locations in relation to the foramen of Monro also were evaluated.

RESULTS: We classified internal cerebral vein branching patterns into 4 types depending on the presence of an extra vessel draining the striatum. Most commonly, the internal cerebral vein continued further as 1 thalamostriate vein (77\%). The lateral direct veins were identified in $22 \%$ of the hemispheres, and usually they terminated at the middle third of the internal cerebral vein (65.45\%). The most common location of the anterior septal vein-internal cerebral vein junction was anterior (57.20\%), with the anterior septal vein terminating at the venous angle.

CONCLUSIONS: Detailed knowledge of the anatomy of the deep cerebral veins is of great importance in neuroradiology and neurosurgery because iatrogenic injury to the veins may result in basal nuclei infarcts. A classification of internal cerebral vein branching patterns may aid clinicians in planning approaches to the third and lateral ventricles.

ABBREVIATIONS: ASV = anterior septal vein; ICV = internal cerebral vein; LDV = lateral direct vein; TSV = thalamostriate vein

$\mathrm{T}$ he internal cerebral vein (ICV), together with the basal vein of Rosenthal and their tributaries, form the deep cerebral venous system. The ICV begins at the union of the anterior septal vein (ASV) and the thalamostriate vein (TSV) at the posterior margin of the foramen of Monro. ${ }^{1}$ Other major tributaries that join the ICV include the lateral direct vein (LDV) and the medial atrial vein. The deep cerebral veins have received little attention in comparison with the cerebral arteries. However, because of their high inter- and intraindividual variability, they are of

Received April 10, 2019; accepted after revision July 25

From the Department of Anatomy (K.B., M.P.Z., A.M., H.M.A., T.K., J.A.W.), Jagiellonian University Medical College, Krakow, Poland; Seattle Science Foundation (R.S.T.), Seattle, Washington; and Departments of Radiology and Rescue Medicine and Multiorgan Trauma (T.P.), University Hospital, Krakow, Poland.

Please address correspondence to Karolina Brzegowy, Department of Anatomy, Jagiellonian University Medical College, Krakow, Poland, 12 Kopernika St, 31-034 Krakow, Poland; e-mail: karolina.brzegowy@gmail.com

Indicates article with supplemental on-line tables. http://dx.doi.org/10.3174/ajnr.A6200 particular interest to neuroradiologists and neurosurgeons. Detailed anatomic knowledge of both the normal and variant anatomy of the ICV branching patterns is crucial to develop optimal surgical strategies to access the third and lateral ventricles, and the large number of diagnostic head studies available currently allows us to conduct an assessment of anatomic variations on a great number of subjects.

The thalamostriate vein is usually the largest tributary of the ICV. The TSV receives several transverse caudate veins, and overall, it collects blood from the caudate nucleus, internal capsule, lentiform nucleus, claustrum, extreme capsule, and the white matter of the frontoparietal lobes. ${ }^{1,2}$ The U-shaped angle where the junction of the TSV and the ICV forms is called the venous angle ${ }^{1}$ and is an anatomic landmark for access to the third ventricle via the lateral ventricle. ${ }^{3,4} \mathrm{~A}$ true venous angle is adjacent to the posterior margin of the foramen of Monro, while a false venous angle lies behind it. ${ }^{3}$ Another important clinical aspect is the ASV termination at the ICV because the location of the ASVICV junction determines the limit of posterior enlargement of 
Table 1: The termination points of the ASV ${ }^{\mathrm{a}}$

\begin{tabular}{llcc}
\hline ASV Termination Type & \multicolumn{1}{c}{ ASV Termination } & \multicolumn{2}{c}{ Total (531 ASVs) } \\
\hline Type A & Venous angle & 383 & $72.13 \%$ \\
Type B & ICV: anterior third & 76 & $14.31 \%$ \\
& ICV: middle third & 52 & $9.79 \%$ \\
& ICV: posterior third & 1 & $0.19 \%$ \\
& ICV elongation (absent TSV) & 20 & $3.77 \%$ \\
& LDV & 5 & $0.94 \%$ \\
& MAV & 15 & $2.82 \%$ \\
& Cavernous sinus & 1 & $0.19 \%$ \\
\hline
\end{tabular}

Note:-MAV indicates medial atrial vein.

${ }^{a}$ Types A and B refer to the classification of ASV-ICV junction proposed by Türe et al (1997), ${ }^{3}$ and they differentiate between the ASV located at the venous angle (anteriorly) or beyond it (posteriorly)."
22), incomplete cross-sections $(n=$ 2), massive hydrocephalus ( $n=3)$, cerebral lesions $(n=14)$, and repeat studies $(n=1)$. The remaining 250 patients were included in this study, of whom 84 were men (33.6\%) and 166 were women (66.4\%).

The study was performed using a multidetector row CT scanner (Optima CT 660; GE Healthcare, Milwaukee, Wisconsin), and a nonionic contrast agent iomeprol (70 $\mathrm{mL}$ administered; Iomeron 350; the foramen of Monro. An ASV terminating at the ICV at the venous angle is a disadvantage, while an ASV joining the trunk of the ICV is an advantage because it allows greater surgical exposure of the third ventricle. Türe et $\mathrm{al}^{3}{ }^{3}$ in 1997, subclassified the true (type I) and false (type II) venous angles further into subtypes: A, in which the ASV-ICV junction is located at the venous angle, and B, in which the ASV joins the main stem of the ICV beyond the venous angle, with a total of 4 venous angle types (IA, IB, IIA, IIB).

The LDV, or the thalamocaudate vein, ${ }^{5,6}$ is located on the floor of the body of the lateral ventricle and enters the ICV at various levels. ${ }^{1}$ A prominent LDV drains blood from the lateral part of the body of the lateral ventricle and receives tributaries from the caudate nucleus. As the LDV takes over the area the TSV usually drains, the TSV may be insufficiently developed when it is present. ${ }^{6,7}$

The increasing use of micro-operative techniques to approach the cerebral areas through which the deep cerebral veins course necessitates a thorough understanding of their course and drainage patterns. An injury to an important deep cerebral vein may result in hemorrhage or basal nuclei infarcts that could lead to severe functional impairment. ${ }^{8}$ The ICV tributaries should be preoperatively investigated via diagnostic imaging studies specifically when these veins would undergo surgical manipulation during access to the third and lateral ventricles.

Hence, the objective of this study was to evaluate the anatomy of the internal cerebral vein and its main tributaries, including the ASV, TSV, and LDV, and to classify the veins depending on their course patterns using CTA.

\section{MATERIALS AND METHODS}

Evaluation of the ICV anatomy in this retrospective study was conducted on adult Polish patients who underwent a head CTA at the Department of Radiology, Department of Rescue Medicine and Multiorgan Trauma, University Hospital in Krakow, Poland, between June 2017 and July 2018. The exclusion criteria included the following: hydrocephalus; cerebral lesions (intracranial hematoma, tumors, vascular malformations); posttraumatic, postsurgical, and poststroke defects that affect the presence and course of the veins; significant imaging artifacts (such as low quality or illegible images); postcraniotomy state; and incomplete cross-sections. Of the initial 292 patients with CTA scans available, a total of 42 were excluded because of significant imaging artifacts $(n=$
$350 \mathrm{mg}$ iodine $/ \mathrm{mL}$; Bracco Imaging, Milan, Italy) was injected. After the bolus reached the common carotid artery at a level of C3-C4, the scanning procedure initiated automatically. The scanner settings were as follows: $120 \mathrm{kV}, 200 \mathrm{~mA}$, and $64 \times 0.625$ $\mathrm{mm}$ section collimation. Axial $0.625-\mathrm{mm}$ slices at increments of $1.25 \mathrm{~mm}$ were reconstructed using a $512 \times 512$ matrix, with a standard kernel applied. The data were analyzed on a dedicated workstation (Advantage Workstation AW4.5; GE Healthcare) equipped with software for MIP and 3D volume-rendering postprocessing of images. The analysis was performed primarily on the MIP cross-sectional images (axial, sagittal, coronal). The statistical analysis was conducted with SPSS, Version 25, 2000-2016 (IBM, Armonk, New York). The $\chi^{2}$ independence test was applied for the dichotomous and nominal variables, and $P$ was set at $<.05$.

The Anatomical Quality Assurance Checklist of the International Evidence-Based Anatomy Working Group, Department of Anatomy, Jagiellonian University Medical College in Krakow, Poland, was used to ensure high-quality reporting of anatomic variants in this study. ${ }^{9}$ The Bioethical Committee of the Jagiellonian University, Krakow, Poland, approved the study (No. 1072.6120.121.2018).

\section{RESULTS}

We evaluated 84 men and 166 women whose average age was $52.3 \pm 16.6$ years (range, 19-89 years). A total of 250 CTA images were deemed eligible for the study.

In the total research material, we observed the following number of ASVs present: no ASV present in 3 hemispheres ( 3 on the right side), 1 present (the most common) in 464 hemispheres (234 right and 230 left hemispheres), 2 present in 32 hemispheres (13 right, 19 left), and last, the least common, 3 ASVs in just 1 hemisphere (left). The number of ASVs that predominated in both right and left hemispheres among patients of both sexes was 1 (95.24\% of right and $91.67 \%$ of left male hemispheres, and $92.77 \%$ right and $92.17 \%$ left female hemispheres). No statistically significant differences were found between the number of ASVs present and the hemispheres in the sample overall $(P=.16)$, in the male patients $(P=.27)$, in the female patients $(P=.36)$, or while comparing the variant presence of the ASV in the different hemispheres and both sexes $(P=.16)$. The terminations of the ASV encountered in the study material is presented in Table 1. The most common was termination at the venous angle $(71.15 \%$ of the right and $73.06 \%$ of the left hemispheres). 


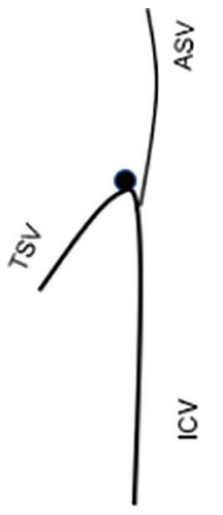

IA) $57.2 \%$

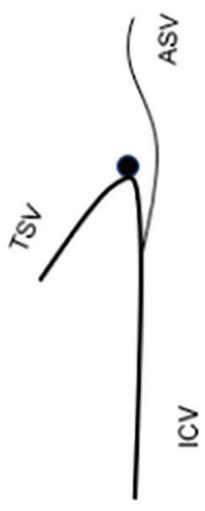

IB) $19.8 \%$

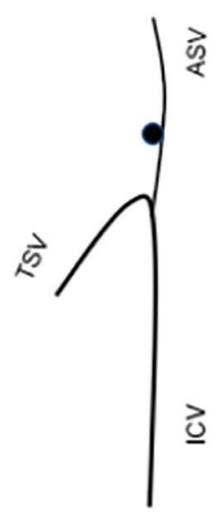

IIA) $20.2 \%$

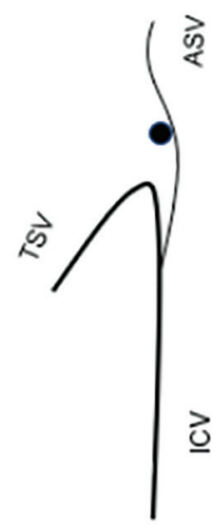

IIB) $2.4 \%$

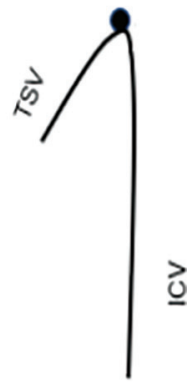

III) $0.2 \%$

FIG 1. Variations of the ASV-ICV junction and its relation to the foramen of Monro. There are a total of 5 venous angle types (IA, IB, IIA, IIB, III). ${ }^{3}$ Types I and II refer to the relation of the venous angle to the foramen of Monro (adjacent to or located behind, respectively), whereas types $A$ and B differentiate the ASV located at the venous angle (anteriorly) or beyond it (posteriorly). In type III, the ASV is absent.

Table 2: The termination points of the lateral direct vein ${ }^{\mathrm{a}}$

\begin{tabular}{clcr}
\hline Branching Type & \multicolumn{1}{c}{ LDV Termination } & \multicolumn{2}{c}{ Total (110 LDV) } \\
\hline Type 2 (a suprathalamic LDV) & ICV: anterior third & 8 & $7.27 \%$ \\
& ICV: middle third & 70 & $63.64 \%$ \\
& ICV: middle third, as a common & 2 & $1.82 \%$ \\
& stem with an MAV & 18 & $16.36 \%$ \\
Type 3 (a retrothalamic LDV) & ICV: posterior third & 8 & $7.27 \%$ \\
& ICV: posterior third, as a common & & \\
& stem with an MAV & 2 & $1.82 \%$ \\
\hline
\end{tabular}

Note:-MAV indicates medial atrial vein.

${ }^{a}$ The presence of the LDV characterizes types 2 and 3 of the ICV branching patterns.
Bifurcation of the TSV was noted in 23 right and 15 left hemispheres with the TSV present.

The LDV was present in 110 hemispheres (62 right, 48 left). No statistically significant differences were found between the presence of the LDV and the hemispheres in the sample overall $(P=.13)$, in the male patients $(P=.47)$, in the female patients $(P=.18)$, or while comparing the variable presence of the LDV in the different hemi-
The ASV-ICV junction was divided into 5 types $^{3}$ and was present as follows: type IA: 286 hemispheres (147 right and 139 left hemispheres); type IB: 99 hemispheres (52 right, 47 left); type IIA: 101 hemispheres (41 right, 60 left); and type IIB: 12 hemispheres (8 right, 4 left) (Fig 1). Type III, ASV absent, was found only in 2 hemispheres (both on the right side). Type IA predominated in both men (50\% of the right and $54.76 \%$ of the left hemispheres) and women $(63.25 \%$ of the right and $56.02 \%$ of the left hemispheres). No statistically significant differences were found between the type of ASV-ICV junction present and the hemispheres in the sample overall $(P=.12)$, in the male patients $(P=.06)$, in the female patients $(P=.28)$, or while comparing the variant presence of the ASV-ICV junction on the different hemispheres and sexes combined $(P=.12)$. Two distinctly different types (asymmetric) of the ASV-ICV junction in both hemispheres were found in 41 men and 89 women.

The number of TSVs found was as follows: no TSVs in 17 hemispheres ( 9 right, 8 left), 1 TSV in 480 hemispheres (239 right, 241 left), and 2 TSVs in only 3 hemispheres (2 right, 1 left). One TSV predominated in both hemispheres in both sexes. No statistically significant differences were found between the number of TSVs present and the hemispheres in the sample overall $(P=$ $82)$, in the male patients $(P=.27)$, in the female patients $(P=.57)$, or while comparing the variant presence of the TSV in the different hemispheres and the sexes combined $(P=.82)$. spheres and sexes combined $(P=.13)$. Only single LDVs were observed. The various points of termination of the LDVs are presented in Table 2. The most common termination point was found to be the middle third of the ICV $(64.52 \%$ of the right and $66.67 \%$ of the left hemispheres).

\section{Classification}

We classified ICV tributaries into 4 types, depending on the main veins draining the basal nuclei (Fig 2). In type 1, only the thalamostriate vein collects blood from the basal nuclei and the LDV is absent. The presence of the LDV located above or behind the thalamus characterizes types 2 and 3, respectively. Type 2 comprises all suprathalamic LDVs terminating at the anterior and middle thirds of the ICV. Type 2 includes retrothalamic LDVs, terminating at the posterior third of the ICV or the great cerebral vein. In type 4 , the basal vein takes an unusual course and drains the basal nuclei. In these hemispheres, the branches from the caudate nucleus join the basal vein, which further forms a loop in the atrium of the lateral ventricle and terminates at the ICV or great cerebral vein. The prevalence of each type is presented in On-line Table 1. Detailed characteristics of deep cerebral veins within types 2 and 3, including the influence of the presence of an LDV on the TSV presence, TSV length, TSV diameter, and the ICV diameter are presented in On-line Table 2. 
Type 1 was the most common type present in this study $(77 \%$ of all hemispheres), while type 4 was present least often ( $1 \%$ of all hemispheres). The same was true for men and women when considered independently. Statistically significant differences were found neither between the hemispheres and the types in general $(P=.23)$ nor between the types and sexes in general $(P=.41)$. Furthermore, statistically insignificant results were also obtained in the presence of the particular types in specific hemispheres

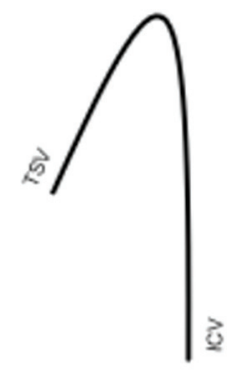

1) $77.0 \%$

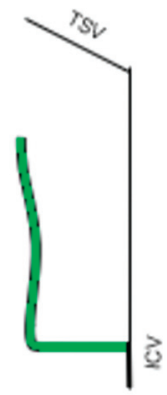

3) $6.0 \%$

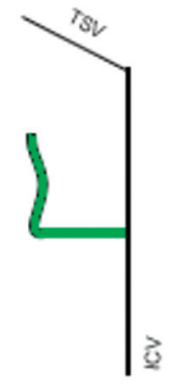

2) $16.0 \%$

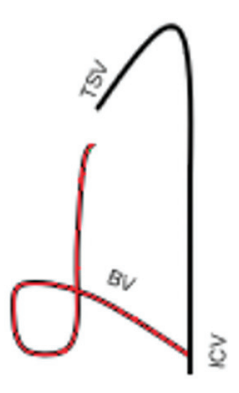

4) $1.0 \%$
FIG 2. Types of ICV branching patterns. We classified ICV tributaries into 4 types with respect to the main veins draining the basal nuclei. In type 1, only the thalamostriate vein collects blood from the basal nuclei. Types 2 and 3 are characterized by the presence of a suprathalamic or retrothalamic LDV, respectively, which receives tributaries from the striatum. In type 4, the basal vein takes an unusual course and drains the basal nuclei.
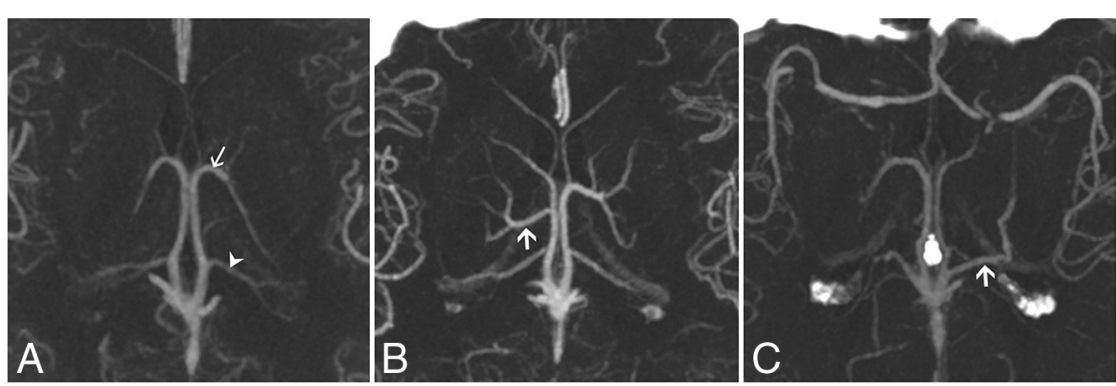

FIG 3. CTA MIP axial cross-sections of the ICV branching patterns. A, Normal ICV anatomy (type 1) in both the left and right hemispheres. The arrow indicates the TSV; the arrowhead, the medial atrial vein. $B$, The suprathalamic variants of the lateral direct vein (right hemisphere). The arrow indicates the LDV. Type 1 is present in the left hemisphere. C, The retrothalamic LDV (left hemisphere). The arrow indicates the LDV. Type 1 is shown in the right hemisphere. only in men $(P=.67)$ or women $(P=.24)$. Last, no statistically significant differences were found in the combined analysis of sex, hemisphere, and type $(P=.25)$. Symmetric types were found between the 2 hemispheres in 50 men and 110 women (59.5\% and $66.3 \%$, respectively). Notwithstanding, the discrepancies were not statistically significant $(P=.29)$.

\section{DISCUSSION}

Previous studies on the deep cerebral veins have focused on the venous anatomy only in the region of the foramen of Monro, and currently existing classifications focus solely on the location of the ASV-ICV junction. ${ }^{7,10,11}$ Numerous researchers have reported differences in ICV branching patterns but did not classify them. ${ }^{10,12}$ This study describes variations in the major veins joining the entire trunk of the ICV and classifies them. It is vital to explore detailed anatomic characteristics of all the tributaries of the ICV, especially the LDV, because a surgical strategy may require altering when it is present.

The primary tributary of the ICV is either the TSV or the LDV. Our study found that the TSV was the only tributary draining the striatum in $77 \%$ of the hemispheres; thus, we consider this type to be a normal anatomy (type 1) (Fig 3A). We found an LDV in $22 \%$ of the hemispheres, which is less than reported previously in a cadaveric study (35\%) and a radiologic study on MRA (36\%). ${ }^{6,7}$ In $18.6 \%$ of the hemispheres included in our study, both the TSV and the LDV drained the striatum. When the LDV was present, the TSV had a reduced diameter compared with the ipsilateral TSV in $38.95 \%$ of the hemispheres. The literature suggests that the size of the LDV may be proportionally inverse to that of the TSV. ${ }^{6}$ The TSV was absent in $3.4 \%$ of the hemispheres, and the LDV was the only tributary draining the striatum (Fig 4). The LDV may drain most blood for the ICV; therefore, the ICV diameter is visibly smaller anterior to the LDV. Identification of the major tributary of the ICV is of immense importance because a surgical occlusion or an injury to the vein draining the basal ganglia may result in a venous infarction. $^{13}$

Our results show that $64 \%$ of the patients had different types of patterns of ICV tributaries in the left and right hemispheres. Therefore, before a surgical approach in the region of the third and the lateral ventricles, the appearance and size of the ICV tributaries should be investigated for each individual patient.

Another important clinical aspect is the relation of an LDV to the thalamus and the lateral ventricles. ${ }^{1}$ In our study, a suprathalamic LDV (type 2: 16.00\%) was more prevalent than the retrothalamic LDV (type 3: 6.00\%) (Fig 3B, $-C)$. The findings of our study are also valuable for an operation on the lateral ventricles. Removal of a mass located in the body of the lateral ventricle exposes vascular structures localized beneath the 


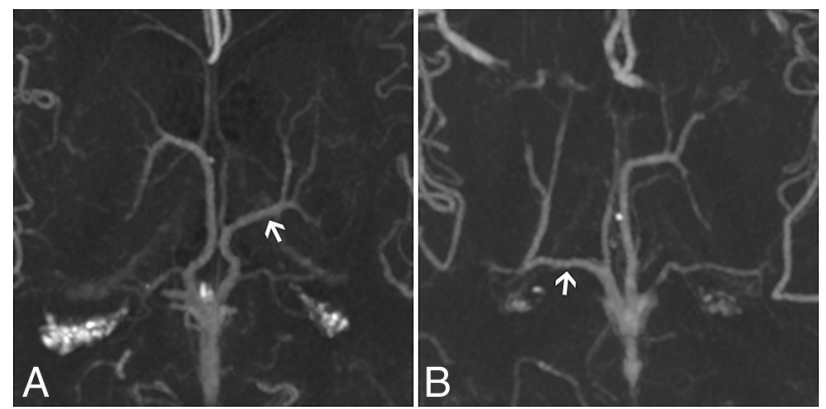

FIG 4. CTA MIP axial cross-sections of the ICV branching patterns with an LDV and an absent thalamostriate vein. Arrows indicate LDVs. $A$, The suprathalamic LDV (type 2; left hemisphere). $B$, The retrothalamic LDV (type 3; right hemisphere).

lesion, and an LDV originating from the anterior or middle portion of the ICV courses farther in this area. Preoperative assessment of veins coursing in the body of the lateral ventricle would help in the development of a dissection plan that minimizes the risk of vascular complications. ${ }^{14}$

As stated above, the anatomy of the ICV tributaries is particularly important in minimally invasive procedures such as deep brain stimulation because placing an electrode properly ensures that no vessel is injured. It has been proved that a minor change in the deep brain stimulation electrode location may make a major difference between penetrating or avoiding a vessel; thus, it can lead to an important difference for the patient's outcome. ${ }^{15,16}$ Furthermore, the venous anatomy of the brain is of great importance to neuroradiologists who perform endovascular interventions in deep brain AVMs and dural arteriovenous fistulas.

An operation on the third ventricle poses a major challenge for neurosurgeons because it is surrounded by vital neural and vascular structures. The foramen of Monro serves as a natural opening from the lateral ventricle to the anterior and middle portions of the third ventricle. ${ }^{6,17}$ However, when a lesion has not widened the foramen of Monro, it can be enlarged surgically as far as the ASV-ICV junction. ${ }^{11,17}$ Successful application of approaches to the third ventricle depends on the surgeon's knowledge of the venous variations of this region. ${ }^{18}$

Our study concluded that the anterior location of the ASVICV junction (type IA: $58.8 \%$ of the hemispheres) is more common than its posterior location (types IB + IIA + IIB: $40.4 \%$ of the hemispheres). These findings are consistent with those of other radiologic studies. Two MRA studies found type IA in $53.9 \%$ and $63.4 \%$ of hemispheres, ${ }^{11,14}$ while a cadaveric study found it in $52.2 \%$ of the hemispheres. ${ }^{3}$ Furthermore, our study revealed that nearly half of the patients (48\%) had an ASV-ICV junction asymmetry between 2 hemispheres. This is particularly clinically important in planning a third ventricle operation because the ventricle can be accessed more easily via the side on which the ASV-ICV junction is located posteriorly. In the event of bilateral ASV-ICV junctions located at the venous angles, posterior enlargement of the foramen of Monro is prevented on both sides and the third ventricle has to be accessed via the interforniceal approach. ${ }^{19}$ Preoperative assessment of the location and symmetry of the ASV-ICV junction may facilitate developing optimal surgical strategies to access the third ventricle and decrease the risk of iatrogenic injury.

Our study was limited by the technique with which the ICV evaluation was conducted. CTA may not have detected small veins terminating at the ICV because of insufficient resolution of this imaging method. However, our goal in this study was to provide general information on the largest of the ICV tributaries as well as their frequencies. Several studies have been conducted on high-field (3T, 9.4T), SWI MRA that focused on the small deep medullary veins. ${ }^{17,20}$ Second, we performed only a qualitative analysis of vessel diameters by comparing the LDV with the ipsilateral TSV and 1 TSV with the contralateral one. Last, we analyzed only white patients; thus, no interracial differences were investigated. Future studies should attempt to investigate the morphometric lengths of the veins to enhance patient outcomes.

\section{CONCLUSIONS}

We found that the TSV alone was the main ICV tributary draining the striatum in $77 \%$ of the hemispheres; therefore, we considered this pattern the normal anatomy (type 1). The LDV as the additional vein draining the striatum was present in $18.6 \%$ of the hemispheres and replaced the TSV in 3.4\%. The ASV-ICV junction usually was located at the foramen of Monro (57.2\%). We also developed a new classification system of the deep cerebral veins draining into the ICV to increase the safety and quality of treatment and diagnosis provided by neuroradiologists and neurosurgeons worldwide.

- Types 2 and 3 warrant consideration during surgical approaches to the body of the lateral ventricle because a sacrifice of a major vein draining the basal nuclei may result in serious complications, such as venous infarction.

- A suprathalamic LDV (type 2) and a retrothalamic LDV (type 3) outline the superior and posterior surfaces of the thalamus, respectively, and require attention during a thalamic operation.

- Type 4 should be identified prior to managing lesions situated in the atrium of the lateral ventricle.

A thorough preoperative understanding of the variation in the patterns of deep cerebral veins may help reduce the risk of iatrogenic injury to those vessels.

Disclosures: Michat P. Zarzecki-UNRELATED: Grants/Grants Pending: Polish Ministry of Science and Higher Education, Comments: "The Best of the Best! 3.0" grant for the young researchers to assist with costs of international competitions and conferences.* Tadeusz Popiela-UNRELATED: Employment: Specjalistyczna Praktyka Lekarska. *Money paid to the institution.

\section{REFERENCES}

1. Salamon G, Huang YP, Michotey P, et al. Deep cerebral veins. In: Salamon G, Huang YP, Michotey P, et al. Radiologic Anatomy of the Brain. Berlin: Springer-Verlag; 1976:210-61

2. Zhang X, Li J, Wen X, et al. Susceptibility-weighted imaging of the anatomic variation of thalamostriate vein and its tributaries. PLOS One 2015;10:e0141513 CrossRef

3. Türe U, Yaşargil MG, Al-Mefty O. The transcallosal-transforaminal approach to the third ventricle with regard to the venous variations in this region. J Neurosurg 1997;87:706-15 CrossRef Medline 
4. Sindou M. The "dangerous" intracranial veins. In: Sindou M. Practical Handbook of Neurosurgery. Vienna:Springer-Verlag; 2009: 71-83

5. Sindou M, Auque J. The intracranial venous system as a neurosurgeon's perspective. Adv Tech Stand Neurosurg 2000;26:131-216 Medline

6. Ono M, Rhoton AL, Peace D, et al. Microsurgical anatomy of the deep venous system of the brain. Neurosurgery 1984;15:621-57 CrossRef Medline

7. Wang J, Wang J, Sun J, et al. Evaluation of the anatomy and variants of internal cerebral veins with phase-sensitive MR imaging. Surg Radiology Anat 2010;32:669-74 CrossRef Medline

8. Sindou MP, Auque J, Jouanneau E. Neurosurgery and the intracranial venous system. In: Yonekawa $Y$, Sakurai $Y$, Keller E, et al eds. New Trends of Surgery for Stroke and its Perioperative Management. Vienna: Springer-Verlag; 2005:167-75

9. Tomaszewski KA, Henry BM, Kumar Ramakrishnan P, et al. Development of the Anatomical Quality Assurance (AQUA) Checklist: guidelines for reporting original anatomical studies. Clin Anat 2017;30:14-20 CrossRef Medline

10. Mokrohisky JF, Paul RE, Lin PM, et al. The diagnostic importance of normal variants in deep cerebral phlebography. Radiology 1956;67:34-47 CrossRef Medline

11. Fujii S, Kanasaki Y, Matsusue E, et al. Demonstration of cerebral venous variations in the region of the third ventricle on phase-sensitive imaging. AJNR Am J Neuroradiol 2010;31:55-59 CrossRef Medline
12. Lang J. Surgical anatomy of the hypothalamus. Acta Neurochir (Wien) 1985;75:5-22 CrossRef Medline

13. Elhammady MS, Heros RC. Cerebral veins: to sacrifice or not to sacrifice, that is the question. World Neurosurg 2015;83:320-24 CrossRef Medline

14. Çagatay Çimsit N, Türe U, Ekinci G, et al. Venous variations in the region of the third ventricle: the role of MR venography. Neuroradiology 2003;45:900-04 CrossRef Medline

15. Nowinski WL, Chua BC, Marchenko Y, et al. Three-dimensional reference and stereotactic atlas of human cerebrovasculature from 7 Tesla. Neuroimage 2011;55:986-98 CrossRef Medline

16. Mahvash M, Pechlivanis I, Charalampaki P, et al. Visualization of small veins with susceptibility-weighted imaging for stereotactic trajectory planning in deep brain stimulation. Clin Neurol Neurosurg 2014;124:151-55 CrossRef Medline

17. Chen Z, Qiao H, Guo Y, et al. Visualization of anatomic variation of the anterior septal vein on susceptibility-weighted imaging. PLoS One 2016;11:e0164221 CrossRef Medline

18. Schijman E. Microsurgical anatomy of the transcallosal approach to the ventricular system, pineal region and basal ganglia. Childs Nerv Syst 1989;5:212-19 CrossRef Medline

19. Greenberg MS. Handbook of Neurosurgery. 7th ed. New York: Thieme Medical Publishers; 2010:168-72

20. Budde J, Shajan G, Hoffmann J, et al. Human imaging at 9.4 T using $\mathrm{T}^{*}{ }^{*}$-, phase-, and susceptibility-weighted contrast. Magn Reson Med 2011;65:544-50 CrossRef Medline 